\title{
Hemorragia rectocolónica aguda masiva secundaria a colitis ulcerosa asociada a lipodistrofia mesentérica colónica*
}

\author{
Drs. ROBERTO R. MANSON ${ }^{1}$, HUGO A. AMARILLO ${ }^{1}$, ROXANA KATSINI B. ${ }^{1}$, \\ MARCELO R. AMARILLO ${ }^{2}$, SEBASTIÁN FOURCANS ${ }^{1}$, HUGO R. AMARILLO ${ }^{1}$ \\ Departamento de Cirugía. \\ 2 Departamento de Radiología. \\ Sanatorio Modelo Universidad Nacional de Tucumán. Tucumán, Argentina.
}

\begin{abstract}
Mesenteric lipodystrophy associated to ulcerative colitis. Case report

Mesenteric lipodystrophy is part of a spectrum of idiopathic primary inflammatory and fibrotic processes that affect the mesentery. Pathophysiologically, these processes may affect the integrity of the gastrointestinal lumen and mesenteric vessels by a mass effect. We report a 64 year old male with an ulcerative colitis who consulted in the emergency room due to hematochezia. The computed tomography revealed a marked mesenteric thickening and colon and rectal wall lesions. The patient was subjected to a proctocolectomy with a good postoperative evolution.
\end{abstract}

Key words: Mesenteric lipodystrophy, ulcerative colitis, hematochezia.

\section{Resumen}

La lipodistrofia mesentérica es una enfermedad inflamatoria crónica inespecífica progresiva que afecta el tejido adiposo del intestino delgado (mesenterio) o del mesocolon. Su etiología es idiopática y su fisiopatología permanece incierta. La lipodistrofia mesentérica con compromiso colorrectal es una condición muy poco frecuente y su asociación a colitis ulcerosa es aún más rara. No hemos encontrado ningún reporte previo en la literatura. Se comunica un caso de lipodistrofia colorrectal asociada a colitis ulcerosa que se presentó con sangrado digestivo bajo agudo masivo.

Palabras clave: Lipodistrofia, mesenteritis, intestino, colon, hemorragia.

\section{Introducción}

La lipodistrofia mesentérica es una enfermedad inflamatoria inespecífica del mesenterio con curso crónico, afectando el tejido graso del intestino del- gado o del colon y recto con sus respectivos mesos. Esta condición provoca un engrosamiento indefinido del tejido adiposo, comprometiendo principalmente al intestino delgado ${ }^{1,2}$.

La paniculitis intestinal fue descrita por Juran

*Recibido el 2 de Febrero de 2009 y aceptado para publicación el 4 de Marzo de 2009.

Correspondencia: Dr. Hugo Amarillo

Laprida 544 (4000). Tucuman, Argentina. FAX +54381-4308408.

E-mail: hugoamarillo@gmail.com 
por primera vez (1924) como una Mesenteritis Retráctil. Crane, en 1955, reportó 5 casos con cambios histológicos. En 1960, Orden llamó a este desorden Paniculitis Mesentérica, denominación aceptada hasta la actualidad ${ }^{1,3-5}$.

El compromiso del colon y del mesorrecto tiene una incidencia baja en esta condición (3-5\%). Su asociación con colitis ulcerosa es aún más rara y no encontramos ningún caso descrito en la literatura hasta la fecha ${ }^{4}$.

El objetivo de esta comunicación es reportar un caso de lipodistrofia colorrectal asociada a colitis ulcerosa que se presentó como una hemorragia digestiva baja aguda masiva.

\section{Caso clínico}

Se describe el caso de un paciente varón de 64 años de edad, con una historia de 20 años de colitis ulcerosa sin tratamiento, que se presentó en la sala de emergencia con shock hemodinámico e inestabilidad tensional debido a un cuadro de hemorragia digestiva baja. Al examen físico inicial, se palpó una masa abdominal en el cuadrante abdominal inferior izquierdo.

La tomografía computada, realizada luego de su estabilización inicial con expansores de volumen y fluidos, demostró un engrosamiento notorio del mesenterio al igual que un compromiso difuso de la pared del colon y del recto (signo del peine) (Figura 1).

La condición clínica del paciente permitió la preparación del intestino con polietilenglicol y la posterior realización de una videocolonoscopia que demostró sangrado rectal y colónico difuso activo y signos endoscópicos compatibles con colitis ulcerosa con afectación de todo el colon.

Se decidió intervenir quirúrgicamente, realizándose una proctocolectomía total con una anastomosis ileoanal con pouch ileal en "S" dada la afectación del mesenterio con retracción difusa que no permitió la realización de una "J". Se completó la operación con una ileostomía temporaria (Figuras 2, 3, 4 y 5).

El paciente cursó un postoperatorio favorable, otorgándose el alta hospitalaria al día 7 de su cirugía.

\section{Discusión}

Esta condición afecta mayormente a los varones en la sexta década (2-3:1 $)^{5}$. Durst describe entre los factores relacionados: cirugías previas (apendicectomía, colecistectomía), litiasis biliar, cirrosis, aneurisma de aorta, enfermedad ulcerosa péptica, cáncer gástrico, trauma abdominal y desórdenes inmunológicos ${ }^{3}$.

La historia natural de la enfermedad consiste en tres estadios clínico-histológicos diferentes: fase de degeneración grasa (lipodistrofia mesentérica), fase inflamatoria (paniculitis mesentérica) y fase final de fibrosis grasa (mesenteritis retráctil) ${ }^{1,2,4}$.

Durante la primera fase histológica, ocurre la infiltración de los macrófagos y linfocitos que remplaza el tejido adiposo. Los hallazgos macroscópicos incluyen engrosamiento difuso de la grasa (42\%), y masas abdominales únicas $(32 \%)$ o múltiples $(26 \%)$. El pronóstico es bueno y es factible su resolución espontánea ${ }^{2,3}$.

La paniculitis mesentérica está caracterizada

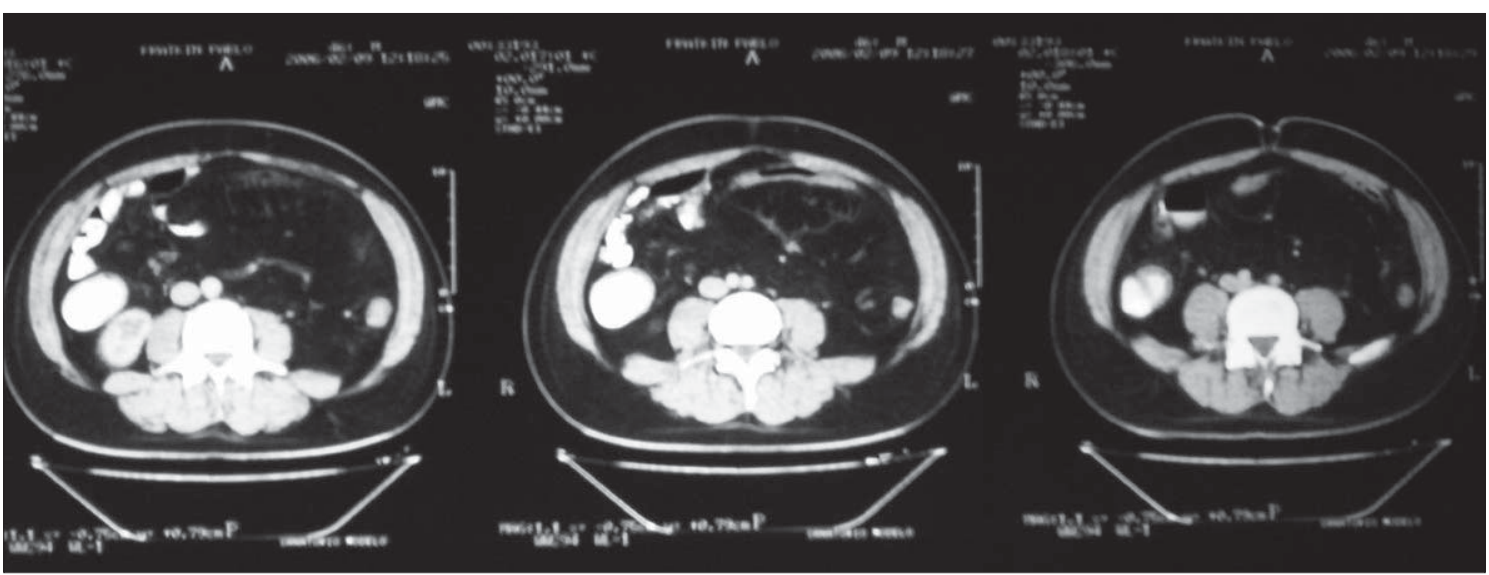

Figura 1. Tomografía computada preoperatoria. 


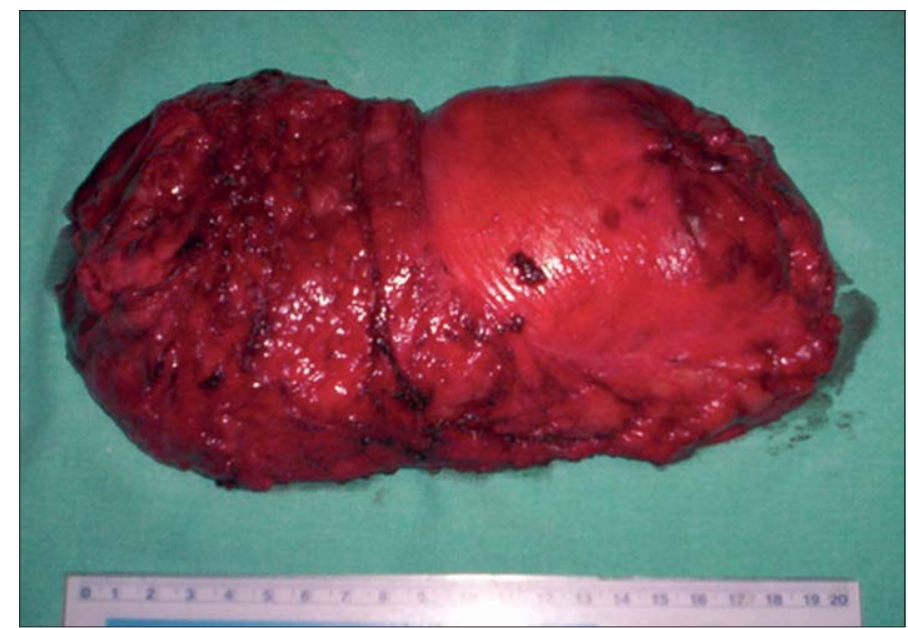

Figura 2. Macroscopia de la resección del recto (lateral).

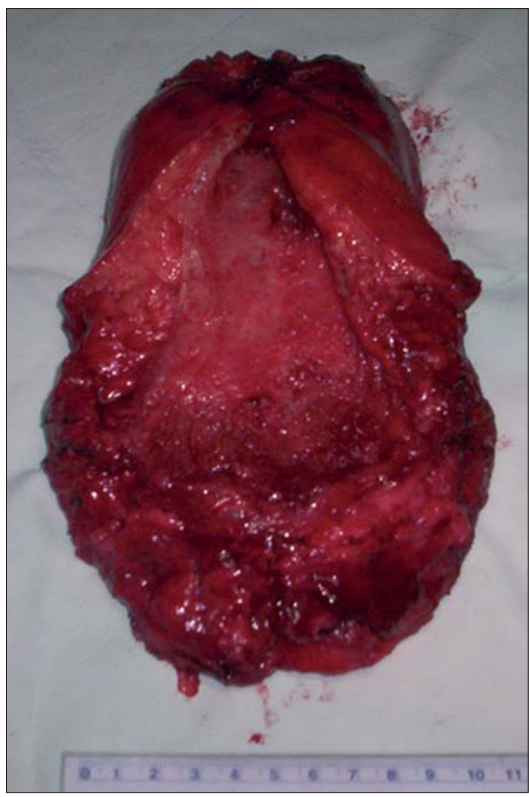

Figura 3. Macroscopia de la resección del recto (frente).

histológicamente por macrófagos espumosos, necrosis focal e infiltración por células inflamatorias. Esta condición afecta el mesenterio del intestino delgado y se presenta sólo ocasionalmente en el mesocolon ${ }^{3}$. Durante la tercera fase, la fibrosis es intensa, remplazando el compromiso inflamatorio, y el resultado final es la mesenteritis retráctil.

Los síntomas son inespecíficos y se relacionan con la forma de presentación. Hallazgos tales como masa abdominal palpable, síndrome febril, anemia, pérdida de peso y diarrea, son habituales. Sin embargo, los pacientes pueden presentar algún tipo de complicación cuando el compromiso colorrectal es prolongado (obstrucción, hemorragia, perforación $)^{2,3}$.

Daskalogiannaki describió su asociación con enfermedades malignas en el $69 \%$ de los casos (linfoma, melanoma y cáncer de mama, pulmón y colon) $)^{4}$.

El diagnóstico es realizado mediante confirmación histológica. El diagnóstico diferencial debe excluir las enfermedades inflamatorias crónicas, infecciosas y neoplásicas.

El tratamiento inicial incluye medidas empíricas y sintomáticas. Cuando los síntomas alteran la calidad de vida o son imposibles de controlar, las drogas como los esteroides, la colchicina, los inmunosupresores y la progesterona se han descrito para el tratamiento del compromiso del intestino delgado. No se han encontrado reportes al respecto con afección del colon.

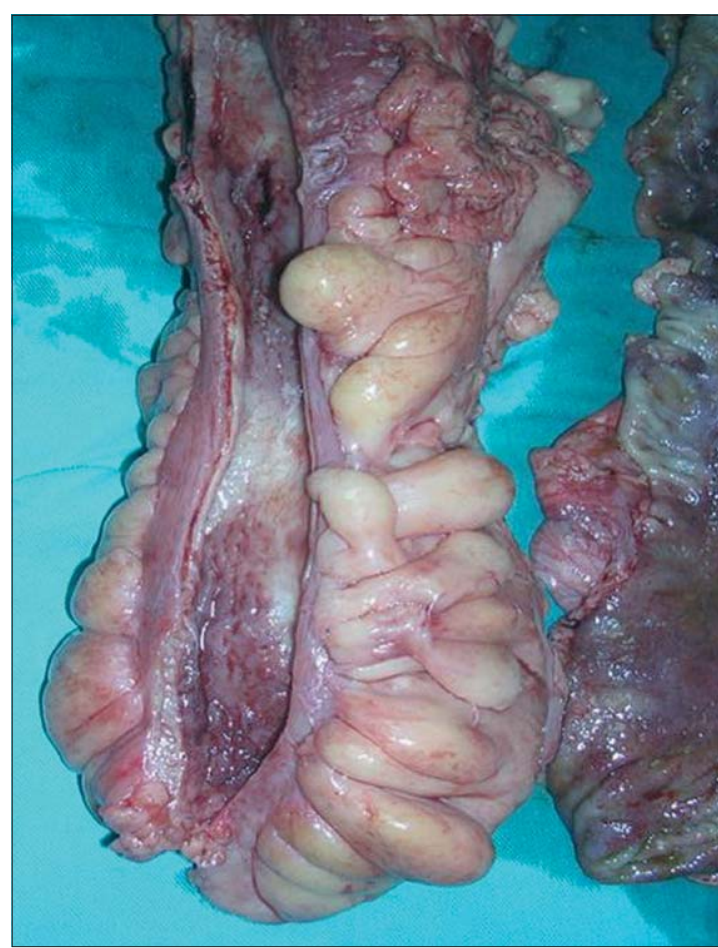

Figura 4. Paniculitis colónica. 
No existe evidencia adecuada en lo que respecta del tratamiento médico en ensayos prospectivos controlados. La cirugía está indicada ante los síntomas obstructivos, la enfermedad complicada y las situaciones de emergencia. Existen comunicaciones previas de regresión completa de la afección sin la realización de procedimiento quirúrgico o terapéutico alguno. La mortalidad es rara ${ }^{2,4,5}$.

\section{Conclusión}

A nuestro parecer, este es el primer caso reportado de lipodistrofia difusa colorrectal asociada a colitis ulcerosa diagnosticada quirúrgicamente luego de una presentación crítica, con curación clínica hasta la fecha.

\section{Referencias}

1. Durst AL, Freund H, Rosenmann E, Birnbaum D. Mesenteric paniculitis: review of literature and presentation of cases. Surgery 1977; 81: 203-211.

2. Wexner SD, Attiyeh FF. Mesenteric panniculitis of the sigmoid colon. Report of two cases. Dis Colon Rectum 1987; 30: 812-815.

3. Kipfer R, Moetel C, Dahlin D. Mesenteric lipodystrophy. Ann Intern Med 1974; 80: 582-588.

4. Adachi Y, Mori M, Enjoji M, Ueo H, Sugimachi K. Mesenteric panniculitis of the colon. Review of literature and report of two cases. Dis Colon Rectum 1987; 30: 962-926.

5. Parra-Dávila E, McKenney MG, Sleeman D, Hartmann R, Rao RK, McKenney K, et al. Mesenteric panniculitis: case report and literature review. Am Surg 1998; 64: 768-771. 